


\title{
Zoonotic helminth parasites of dog in Bishoftu Town, central Ethiopia: prevalence, dog owners' knowledge and control practice
}

\author{
Mitiku Tadesse ${ }^{1}$, Dinka Ayana ${ }^{2}$,Bersisa Kumsa ${ }^{2}$ and Abebe Fromsa ${ }^{2^{*}}$ \\ ${ }^{1}$ Ministry of Agriculture, Livestock and Fisheries Development, SSNNP, Hadiya Zone, Ethiopia \\ ${ }^{2}$ College of Veterinary Medicine and Agriculture, Addis Ababa University, Bishoftu, Ethiopia, \\ P.O. Box 34 . \\ *Corresponding author, e-mail: abebe.fromsa@aau.edu.et or afromsa@yahoo.com
}

\begin{abstract}
Many dog helminth parasites are endemic in many countries of the world posing public health threat. However, they were neglected and less studied in the developing countries such as Ethiopia. This cross-sectional study carried out from November 2016 to April 2017 in Bishoftu town aims at estimating the prevalence of major gastrointestinal tract (GIT) zoonotic helminth parasites of dogs and assessing dog owner's knowledge and control practice against zoonotic dog parasites. Accordingly, the whole area of Bishoftu town was divided into 60 blocks, of which 10 were randomly selected for the study. A structured questionnaire was prepared in English, pretested and administered face to face to 140 dog-owning respondents using two local languages: Amharic and Affan Oromo. Fecal samples collected from 238 dogs after administration of ivermectin and praziquantel at recommended doses to increase sensitivity of detection and to get favorable cooperation of owners. For case detection flotation technique is used for parasite egg identification and parasite morphology for parasites observed in the feces. Pearson's Chi-square ( $\mathrm{x} 2), \mathrm{p}$-values and $95 \%$ confidence intervals calculated to measure association. Four zoonotic helminthic parasites detected with combined infection prevalence of 59.24\% (95\% CI: 52.84-65.35). The prevalence of each was $33.61 \%$ (95\% CI: $27.86-39.90$ ) Ancylostoma (A.) caninum, 29.41\% (95\% CI: 23.93 - 35.56) Toxocara (T.) canis, 19.75\% (95\% CI: 15.14 - 25.34) Dipylidium (D.) caninum, and $2.10 \%$ (95\% CI: $0.87-4.98)$ Echinococcus (E.) granulosus. The prevalence of $A$. caninum and $T$. canis was significantly higher than the prevalence of $D$. caninum and $E$. granulosus. Mixed infection with two parasites recorded in $13.87 \%$ whereas concurrent infection with three parasites registered in $5.88 \%$ of the dogs. Statistically significant association $(p<0.05)$ was observed between overall infection prevalence and the sex of dogs, where infection prevalence of $A$. caninum
\end{abstract}


$(p<0.001)$ and $T$. canis $(p<0.001)$ were significantly higher in male than in female dogs. The questionnaire survey revealed that only $40 \%$ of the respondents were aware of the transmission of zoonotic helminthes to humans while none know the route of transmission. Of all the respondents, $58.57 \%$ reported regular deworming of their dogs, at least twice per annum, whereas $47.86 \%$ of respondents clean and dispose dogs' excrement with household garbage and $88.57 \%$ of dog owners remove dogs' excrement without using glove, facemasks, boots and/or coverall/gown for personal protection In relative terms high prevalence of zoonotic helminth parasites infestation was observed in owned dog population with poor awareness about route of transmission. Thus, it is advisable to create awareness of dog owners in waste management and use of Personal Protective Equipment's (PPE).

Keywords: Ancylostoma; Bishoftu; Dipylidium; Echinococcus; Ethiopia; Toxocara

\section{Introduction}

Man has had a close association with dog since long before the dawn of history (Morey, 1994). In the modern society, dogs are used for diverse purposes such as military functions, guiding the blind, guarding property and being companion often considered as part of the family by households (Westgarth et al., 2013). In Ethiopia, dogs are important animals in many urban and rural households mainly kept as house guards, but also sometimes kept as hunting and companion animals (Hailu et al., 2007). However, despite the benefits of pets to their owners, there are also health hazards to humans. Animal health professionals may also have high risks of exposure to zoonotic diseases including parasites. A questionnaire survey conducted in Australia involving 344 veterinarians revealed $44.9 \%$ contracting a zoonotic disease including parasites during their careers, $63.2 \%$ of these have worked in companion animal practice indicating the high risk of animal health professionals' exposure to dog borne zoonosis (Dowd et al., 2013).

Thus, dogs are responsible for the spread of several zoonotic diseases having serious health consequences to humans. A number of parasitic infections that are capable of transmission from pets to humans include cutaneous larva migrans, visceral larva migrans and ocular migrans caused by Ancylostoma spp. and Toxocara spp. (Gawor et al., 2008; Paul et al., 2009; Bowman et al., 2010; Finsterer and Auer, 2013). Dogs are definitive hosts for a number of cestodes 
including Taenia hydatigena (Cysticercus tenuicollis), Taenia multiceps (Coenurus cerebralis), Taenia ovis (Cysticercus ovis) and Echinococcus granulosus (hydatid cyst) that causes severe economic losses due to downgrading or condemnation of various visceral organs and carcasses of livestock (Macpherson, et al., 2005; Fromsa and Jobre, 2012; Elsheikha and Patterson 2013). Some of these zoonotic parasitic diseases of dogs are very important causes of human mortality and morbidity in developing countries due to the extensive existence of uncontrolled dog population in close proximity to increasing density of human population in both rural and urban environment (Dutta, 2002). Human infection with hydatid cysts can cause serious disease and death (Zajac and Conboy, 2012). The persistent occurrence and transmission of these diseases in the poorer countries attributed to poor level of hygienic conditions, lack of sufficient veterinary attention to parasitic zoonotic diseases of dogs and lack of awareness of the public about the presence and transmission of these diseases (Traub et al., 2002).

Gastrointestinal helminths may also have a serious impact on health of dogs. Parasitized animals may show a variety of symptoms depending on the infesting parasite species and density. Severe cases such as heavy hookworm infestation can cause severe anemia and death due to their voracious blood sucking habits (Barutzki and Schaper, 2003; Zajac and Conboy, 2012). Mild to moderate cases of dog parasitism may impede the successful rearing of dogs due to lowered resistance to infectious diseases, retarded growth, reduced work and feed efficiency, and general ill health (Little et al., 2009).

Several research reports have documented the prevalence of canine intestinal parasites in various locations around Ethiopia and the world (Hailu et al., 2007; Little et al., 2009; Zewdu et al., 2010; Joffe et al., 2011; Abere et al., 2012; Getahun and Addis, 2012; Gebreselasie et al., 2013). However, due to regional variations in parasite prevalence, such information is often of limited value outside the specific study areas. Therefore, to devise a successful control program against any disease of public health importance, understanding epidemiology of the diseases as well as knowledge, practice and attitude of the community with regard to disease transmission route is crucial (Macpherson, 2005). Identification of the knowledge gap and increasing the awareness and knowledge of the community based on the observed gap and deficiency is considered reliable for making informed intervention that will result in changing attitudes and practices of the society to minimize disease burden. 
However, there is lack of sufficient information about the magnitude of zoonotic parasitic disease prevalence among dogs. Little is known about the awareness and practice of the dog-owning community of Bishoftu area with regard to the practice of regular dog deworming, prevention of the communal environment from contamination by zoonotic parasite laden dog fecal material and self-protection against potential zoonotic parasitic infection from their dogs (Hailu et al., 2007; Zewdu et al., 2010; Merga and Sibhat, 2015). To fill the existing information and knowledge gap, all relevant data and information should be collected including the prevalence of zoonotic parasites in dogs' feces and habitual practices of dog owners in the management of their dog's excreta. Therefore, this study conducted with specific objective to estimate the prevalence of major zoonotic helminth parasites in the feces of dogs and to assess the knowledge and practices of dog owners in Bishoftu Town.

\section{Materials and methods}

\section{The study area}

The study was conducted from November 2016 to April 2017 in Bishoftu town located southeast at a distance of 47.9 Kilometers away from the capital, Addis Ababa. The town of Bishoftu is situated at an elevation of 1978 meters above sea level and $8^{\circ} 35^{\prime} \mathrm{N}$ latitude and $39^{\circ} 06^{\prime} \mathrm{E}$ longitude experiencing a bimodal pattern of rainfall. The main rainy season extends from June to September contributing for $84 \%$ of the expected annual rainfall. A short rainy season occurs from March to May with an average annual rainfall of $800 \mathrm{~mm}$. The mean annual minimum and maximum temperatures are $12.3^{\circ} \mathrm{C}$ and $27.7^{\circ} \mathrm{C}$, respectively, with an overall average of $18.7^{\circ} \mathrm{C}$. The mean relative humidity is $61.3 \%$ (Mengistu et al., 2002).

\section{Study design and area selection}

The study design is cross-sectional. Google map of Bishoftu town downloaded from Google website: http://earth.google.com/streetview/. Then then the whole area of the town divided into non-overlapping approximately equal-sized blocks surrounded by wide asphalt or cobble stone roads on the map. Then each equal-sized adjacent block was marked using four different colors: green, red, yellow and light blue (Fig.1A). Accordingly, the whole of Bishoftu town divided into 60 blocks, each adjacent area having approximately equal size and marked with one of the four colors producing 15 blocks marked by each 
color. The four colors were written on a piece of paper and entered for a pick by lottery system. By the lottery system, the green color was selected. Since there were 15 green color marked blocks of Bishoftu town and limited capacity to cover all, 10 blocks reselected randomly by the lottery system (Fig. 1B). The selected areas were then visited to mark their boundaries on the ground using turning roads and fixed permanent posts such as hotels, buildings, and long fences as markers. Then all the households within the marked boundaries of the selected blocks that are having one or more dogs were identified, the number of owned dogs counted and color marks were put on the outer door or fence of the dog-owning households (Fig.2). Accordingly, from 469 households located within the selected blocks, 334 households were marked as dog-owning households.
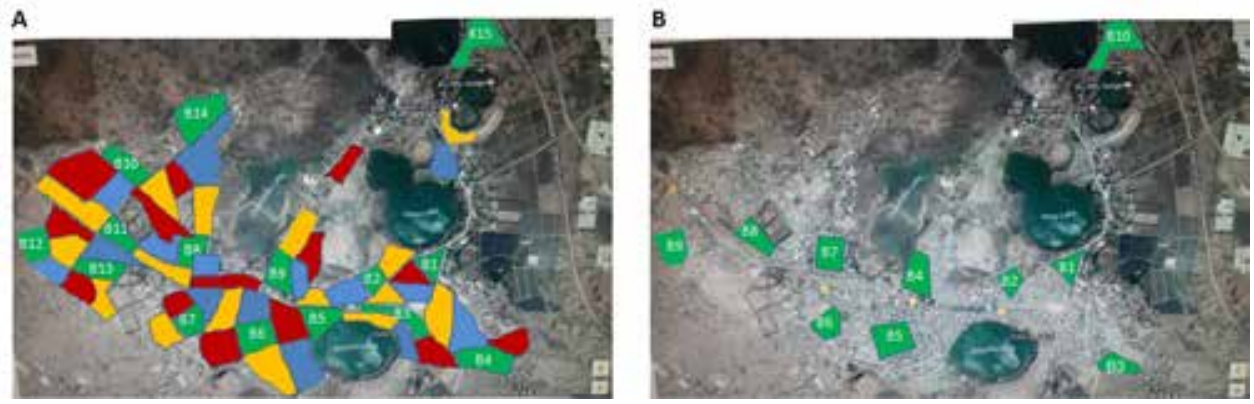

Fig.1: Map of Bishoftu town, A) map divided into 60 approximately equal-sized non-overlapping blocks marked with four different colors. B) The randomly selected 10 green colored blocks and included in the study.

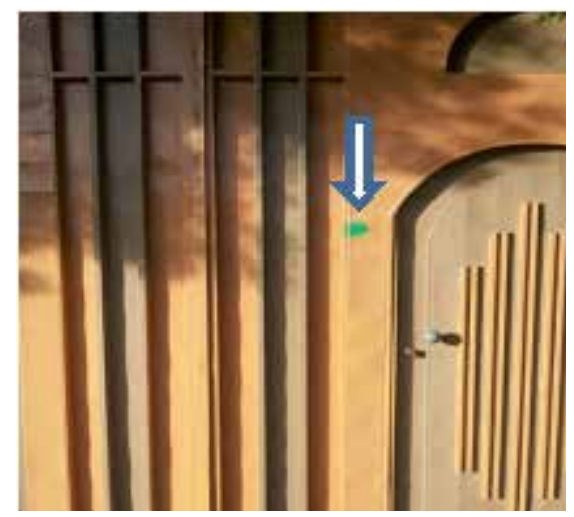

Fig.2: The outer fence door of dog-owning household marked with green color (open arrow) for ease of differentiating it from other households that do not have dog. 


\section{Fecal sample collection}

Each dog-owning household identified by color marks were visited and owners were explained about the study and asked if they could agree to participate in the study and get their dogs dewormed with ivermectin at $200 \mathrm{\mu g} /$ $\mathrm{kg}$ and praziquantel at a dose rate of $10 \mathrm{mg} / \mathrm{kg}$ as an incentive to them and as a supplementary diagnostic tool for detection of helminth infection. When the households agree, then a dog is given the treatments based on estimated weight and fecal samples were collected the next day in the morning. The variables studied include age and sex recorded with similar identification numbers on both the sample vials and recording sheets. For the purpose of analysis, dogs were classified in to three groups based on age difference as puppies ( $\leq$ 6 month), young (6 months to 1 year) and adult ( $\geq 1$ year of age) as applied by Zewdu et al., (2010).

From a total of 469 households registered within the 10 randomly selected blocks, 334 were dog-owning and all were considered for inclusion provided consent is given and collection of fecal sample is possible after deworming. Finally, fecal sample collection made possible only from 238 dogs. The remaining excluded since obtaining fecal sample was impossible either due to lack of owners' interest to participate or failure to submit fecal sample after deworming. For the 238 household dogs, the expelled adult parasites (Fig. 3d) and/ or approximately 6 grams of fresh fecal samples collected, either immediately after voided from target animal or directly from the rectum. Then, the samples labeled, preserved in 10\% formalin and transported to Parasitology Laboratory of College of Veterinary Medicine and Agriculture, Addis Ababa University for processing.

\section{Laboratory test}

At the laboratory, the fecal samples were examined using flotation techniques in saturated sodium chloride ( $\mathrm{NaCl}$; $\mathrm{SG} 1.18-1.20$; to identify the eggs of nematodes) and zinc sulphate (ZnSO4; SG 1.20 or sucrose solution (SG 1.27) for cestode egg detection (Mirzaei and Fooladi, 2013). Three grams of the sample measured and added to mortal then $42 \mathrm{ml}$ of floatation fluid added to fecal sample contained in mortar then crushed well with pestle and mixed thoroughly. The solution sieved by using tea strainer into beaker to remove rough materials. The filtrate added to centrifuge tube and centrifuged at $3000 \mathrm{rpm}$ for 3 minutes, then a top-up of floatation fluid was added until a cone shape is 
formed at the top of the centrifuge tube, then coverslip was placed on the top of the tube and allowed to stand for 15-20 minute. The coverslip raised up gently and placed on the microscopic slide. The sample is positive when an adult parasite expelled and/or when at least one egg of the specific parasite is detected (Lorenzini et al., 2007). The eggs were identified by observing the slides under 10x magnification of compound microscope using ova identification keys to the level of genera or species based on the key provided by Hendrix (2003) and adult parasites were identified based on the keys provided by Zajac and Conboy, (2012). Only the presence or absence of a given parasite is assessed to calculate prevalence; neither egg nor adult parasite count was performed. When an egg and/or adult parasite is seen, the case is recorded as positive, when both egg and adult parasite of any species is absent, the case is recorded as negative. For D. caninum, all case detections were based on morphologic characteristics of expelled adult parasites, except in two dogs in which eggs were also seen in fecal floatation.

\section{Questionnaire survey}

A structured questionnaire was prepared to gather information on dog ownership, feeding, cleaning and deworming practices, the extent of awareness on zoonotic parasites of dogs, their transmission routes to human, and other related factors (Table 4). The questionnaire developed in English was translated either to Amharic or Oromifa and administered by members of the research team to gather information on dog ownership; feeding, cleaning and deworming practices; knowledge on zoonotic parasites' routes of transmission and other related factors. The questionnaire administered in Amharic and Oromifa then translated back to English to record. The questionnaire was pretested before administration via a face-to-face interview with adult person responsible for the care of the $\mathrm{dog} / \mathrm{s}$ at the household. The questionnaires included in the study analysis were those administered to 140 dog-owning residents, who gave their consent to participate and answered all questions.

\section{Data Management and Analysis}

The raw data were first entered into Microsoft excel database system and imported to STATA statistical software version 13 (Stata Corp. 2013) for computation of descriptive statistics such as percent prevalence and frequency distributions. Pearson's Chi-square ( $\mathrm{x} 2$ ) used to measure association between parasite prevalence and age and sex of dogs. In all the analysis, the confidence 
level was held at $95 \%$ and the results were considered significant when p-value was $<0.05$.

\section{Results}

Adult parasites and/or eggs of four intestinal zoonotic parasite species of dogs were identified and 141 (59.24\%), (95\% CI: 52.84-65.35) of dogs had $\geq 1$ species of zoonotic parasite from a total of 238 canine fecal samples examined where the maximum number of parasites detected in mixed infection was three (Table 1, 2). The prevalence of each was $33.61 \%$ (95\% CI: 27.86 - 39.90) A. caninum, $29.41 \%$ (95\% CI: $23.93-35.56)$ T. canis, $19.75 \%$ (95\% CI: $15.14-25.34) D$. caninum, and $2.10 \%$ (95\% CI: $0.87-4.98) E$. granulosus. The prevalence of $A$. caninum and T. canis was significantly higher $(\mathrm{p}<0.001)$ than the prevalence of D. caninum and E. granulosus. Ancylostoma caninum was the most prevalent, while Echinococcus granulosus was the least detected in this study (Table 1).

Table 1: Intestinal helminth parasites of zoonotic importance identified in dog fecal samples based on expelled adult parasites and/or eggs in Bishoftu town

\begin{tabular}{lcccc}
\hline Helminth parasites & $\begin{array}{l}\text { Number } \\
\text { examined }\end{array}$ & $\begin{array}{l}\text { Number (\%) } \\
\text { positive }\end{array}$ & SE (\%) & $\mathbf{9 5 \% ~ C I ~}$ \\
\hline Echinococcus granulosus & 238 & $5(2.10)$ & 0.93 & {$[0.87-4.98]$} \\
Dipylidium caninum & 238 & $47(19.75)$ & 2.59 & {$[15.14-25.34]$} \\
Ancylostoma caninum & 238 & $80(33.61)$ & 3.07 & {$[27.86-39.90]$} \\
Toxocara canis & 238 & $70(29.41)$ & 2.96 & {$[23.93-35.56]$} \\
Total & 238 & $141(59.24)$ & 3.19 & {$[52.84-65.35]$} \\
\hline
\end{tabular}

Mixed infection with two parasites recorded in $13.87 \%$ whereas concurrent infection with three parasites registered in $5.88 \%$ dogs.

Table 2: Prevalence of mixed helminths infection in dogs (dogs harboring zoonotic dog parasite infection with at least one detected parasites).

\begin{tabular}{lcccc}
\hline $\begin{array}{l}\text { Number of parasites per } \\
\text { infested dogs }\end{array}$ & $\begin{array}{l}\text { Number } \\
\text { examined }\end{array}$ & $\begin{array}{l}\text { Number (\%) } \\
\text { Positive }\end{array}$ & SE (\%) & 95\% CI \\
\hline One parasite & 238 & $94(39.49)$ & 3.18 & {$[33.44-45.89]$} \\
Two parasites & 238 & $33(13.87)$ & 2.24 & {$[10.00-18.90]$} \\
Three parasites & 238 & $14(5.88)$ & 1.53 & {$[3.50-9.72]$} \\
Total & $\mathbf{2 3 8}$ & $\mathbf{1 4 1}(\mathbf{5 9 . 2 4})$ & $\mathbf{3 . 1 9}$ & {$[\mathbf{5 2 . 8 4}-\mathbf{6 5 . 3 5}]$} \\
\hline
\end{tabular}


Although parasites detected in fecal samples of every age and sex groups of dogs examined in this study, parasitism more frequently detected in fecal samples of adult $\operatorname{dog} \geq 1$ year of age and in males than in females (Table $3, p<$ 0.001).

Table 3: The prevalence of intestinal zoonotic helminth parasites in different age and sex groups of dogs in Bishoftu town.

\begin{tabular}{|c|c|c|c|c|c|}
\hline Parasites & $\begin{array}{l}\text { Risk } \\
\text { factors }\end{array}$ & $\begin{array}{l}\text { Categories } \\
\text { (Groups) }\end{array}$ & Frequency (\%) & $x^{2}$ & p-value \\
\hline \multirow[t]{2}{*}{ E. granulosus } & Age & $\begin{array}{l}\text { Puppy ( } \leq 6 \text { month) } \\
\text { Young ( } 6 \text { month- } 1 \text { year) } \\
\text { Adult ( } \geq 1 \text { year })\end{array}$ & $\begin{array}{c}0 \\
0 \\
5(2.10)\end{array}$ & 1.7992 & 0.407 \\
\hline & Sex & $\begin{array}{l}\text { Male } \\
\text { Female }\end{array}$ & $\begin{array}{c}5(2.10) \\
0\end{array}$ & 1.1584 & 0.282 \\
\hline \multirow[t]{2}{*}{ D. caninum } & Age & $\begin{array}{l}\text { Puppy } \\
\text { Young } \\
\text { Adult }\end{array}$ & $\begin{array}{c}6(2.52) \\
4(1.68) \\
37(15.55)\end{array}$ & 0.7835 & 0.676 \\
\hline & Sex & $\begin{array}{l}\text { Male } \\
\text { Female }\end{array}$ & $\begin{array}{c}35(14.71) \\
12(5.04)\end{array}$ & 1.9286 & 0.165 \\
\hline \multirow[t]{2}{*}{ A. caninum } & Age & $\begin{array}{l}\text { Puppy } \\
\text { Young } \\
\text { Adult }\end{array}$ & $\begin{array}{c}12(5.04) \\
7(2.94) \\
61(25.63\end{array}$ & 0.3521 & 0.839 \\
\hline & Sex & $\begin{array}{l}\text { Male } \\
\text { Female }\end{array}$ & $\begin{array}{l}56(23.53) \\
24(10.08)\end{array}$ & 10.5988 & 0.001 \\
\hline \multirow[t]{2}{*}{ T. caninum } & Age & $\begin{array}{l}\text { Puppy } \\
\text { Young } \\
\text { Adult }\end{array}$ & $\begin{array}{c}13(5.46) \\
7(2.94) \\
50(21.01)\end{array}$ & 0.3303 & 0.848 \\
\hline & Sex & $\begin{array}{l}\text { Male } \\
\text { Female }\end{array}$ & $\begin{array}{l}48(20.17) \\
22(9.24)\end{array}$ & 11.0208 & 0.001 \\
\hline \multirow[t]{2}{*}{ Overall } & Age & $\begin{array}{l}\text { Puppy } \\
\text { Young } \\
\text { Adult }\end{array}$ & $\begin{array}{c}26(10.92) \\
10(4.20) \\
105(44.12)\end{array}$ & 11.3927 & 0.318 \\
\hline & Sex & $\begin{array}{l}\text { Male } \\
\text { Female }\end{array}$ & $\begin{array}{c}105(44.12) \\
36(15.13)\end{array}$ & 2.2939 & 0.001 \\
\hline
\end{tabular}




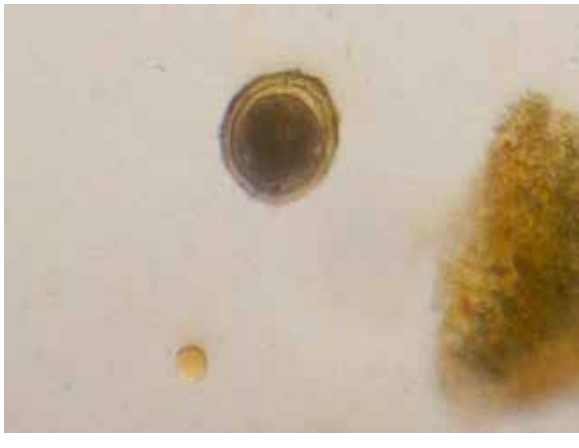

a) Eggs of Toxocara canis detected in the feces of a local dog from Bishoftu (X10)

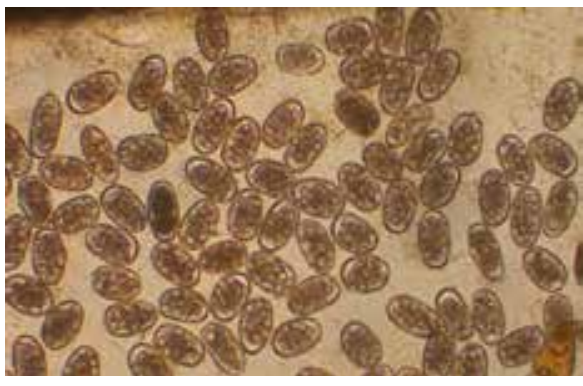

c) Eggs of $A$. caninum, in a single microscopic field, in 2 years old owned dog from Bishoftu town (X10)

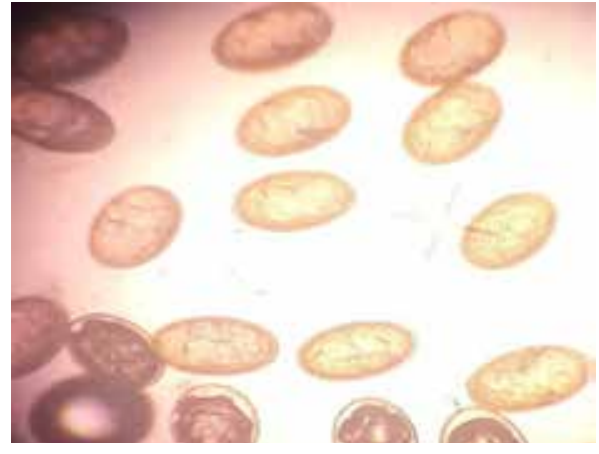

b) Mixed infection with T. caninum and $A$. caninum (X10)
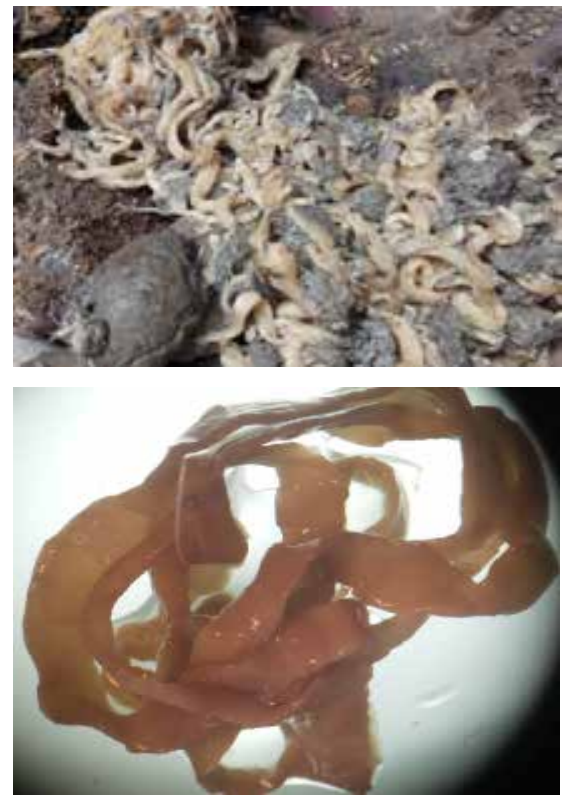

d. Top) D. caninum, purged with feces after praziquantel treatment of 8-month-old local owned dog. Bottom) the parasite viewed under stereomicroscope.

Figure 3: The adult parasites and eggs of zoonotic helminths identified from dogs of Bishoftu. 


\section{Questionnaire survey result}

Ninety percent (90\%) of the respondents keep dogs to guard them against thieves and wildlife whereas the remaining $10 \%$ keep dogs as companion animals. From respondent households, who have dogs, $61.43 \%(86 / 140)$ had a single dog whereas $38.57 \%$ (54/140) had two or more dogs with $60 \%$ of the respondents reporting free roaming of their dogs outside their property. Of all respondents, $73.57 \%$ knew that dogs can have gastro-intestinal parasites and $52.52 \%$ of them have observed parasites in their dog's excrement. The characteristics description given about the observed parasite by $34.29 \%$ of respondents fits to tapeworms' morphology (locally called "Yewusha Koso"). Out of the total respondents, $40 \%$ had information about parasitic diseases transmitted from dogs to human, but none of them has information about routes of exposure for humans. About $37.14 \%$ of the respondents do let their dogs to defecate on the public fields whereas $47.86 \%$ of owners dispose dog's feces with household garbage out of their compound. The questionnaire survey revealed that $88.57 \%$ of dog owners clean and dispose their dogs' excreta with household garbage without using glove, facemasks, boots and/or coverall/gown for personal protection predisposing themselves for potential contamination and infection. Out of all the respondents, $58.57 \%$ reported deworming of their dogs at least twice per year. The assessment of the feeding practice of dog owners revealed that $83.67 \%$ (82/98) of the dog owners either feed uncooked offal from butcher house or back yard slaughter to their dogs or release them to search for their own feed with a potential access to infected raw condemned offal. (Table 4). 
Table 4: Knowledge, and practice of dog owners regarding potential zoonotic parasitic disease of dogs in the Bishoftu town $(\mathrm{N}=140)$.

\begin{tabular}{|c|c|c|c|}
\hline Variable & Number (\%)_respondents & SE (\%) & $95 \% \mathrm{CI}$ \\
\hline \multicolumn{4}{|l|}{ Dog ownership } \\
\hline One dog & $86(61.43)$ & 4.12 & [53.02-69.21] \\
\hline Two and more dogs & $54(38.57)$ & 4.12 & [30.79-46.98] \\
\hline \multicolumn{4}{|l|}{ Purpose of keeping } \\
\hline Guard against theft & $123(87.86)$ & 2.77 & [81.23-92.36] \\
\hline Guard against wild animal & $3(2.14)$ & 1.23 & [0.68-6.51] \\
\hline As companion pet & $14(10.00)$ & 2.54 & {$[5.97-16.27]$} \\
\hline \multicolumn{4}{|l|}{ Housing of dogs at night } \\
\hline In the cage & $47(33.57)$ & 4.00 & [26.16-41.89] \\
\hline In the house & $2(1.43)$ & 1.00 & {$[0.35-5.62]$} \\
\hline With the children & $2(1.43)$ & 1.00 & {$[0.35-5.62]$} \\
\hline Roam in and outside of the compound & $84(60)$ & 4.15 & [51.57-67.86] \\
\hline In the compound only & $5(3.57)$ & 1.57 & {$[8.37-8.37]$} \\
\hline \multicolumn{4}{|l|}{ Feeding of $\mathrm{dog} / \mathrm{s}$} \\
\hline Uncooked offal/head from butcher house & $15(10.71)$ & 2.62 & {$[6.52-17.10]$} \\
\hline Uncooked offal/head from backyard slaughter & $5(3.57)$ & 1.57 & {$[1.47-8.37]$} \\
\hline Left over from family dish & $23(16.43)$ & 3.14 & [11.11-23.61] \\
\hline From all of the above three sources & $85(60.71)$ & 4.14 & [52.23-68.53] \\
\hline Roam and gets its own feed & $6(4.29)$ & 1.71 & [1.91-9.23] \\
\hline From all above sources & $6(4.29)$ & 1.71 & [1.91-9.23] \\
\hline \multicolumn{4}{|l|}{ Usual place of defecation of $\mathrm{dog} / \mathrm{s}$} \\
\hline In public field & $52(37.14)$ & 4.09 & [29.45-45.53] \\
\hline In their cage only & $32(22.86)$ & 3.56 & [16.57-30.63] \\
\hline In the compound & $39(27.86)$ & 3.80 & [20.98-35.95] \\
\hline In all of the above & $17(12.14)$ & 2.77 & {$[7.64-18.76]$} \\
\hline \multicolumn{4}{|l|}{ Way of dogs' excrement cleaning and disposal } \\
\hline Clean and bury in the ground & $8(5.71)$ & 1.96 & {$[2.85-11.09]$} \\
\hline Clean and add to the toilet & $28(20.00)$ & 3.34 & [14.11-27.54] \\
\hline Clean and dispose with house garbage & $67(47.86)$ & 4.23 & [39.61-56.21] \\
\hline Do not clean & $37(26.43)$ & 3.74 & {$[19.71-34.44$} \\
\hline \multicolumn{4}{|l|}{ Use of protective equipment during cleaning } \\
\hline Uses glove, facemasks boots and/or coverall/gown & $16(11.43)$ & 2.69 & [7.07-17.93] \\
\hline No use of glove, facemasks, boots and/or coverall/gown & $123(88.57)$ & 2.69 & [82.06-92.92] \\
\hline \multicolumn{4}{|l|}{ Owners viewpoint towards dog parasites } \\
\hline Knows that dogs can have parasites & $103(73.57)$ & 3.74 & [65.55-80.28] \\
\hline Observed a parasite in their dogs' excrement & $73(52.52)$ & 4.25 & [44.12-60.77] \\
\hline Know parasites transmitted from dogs to humans & $56(40.00)$ & 4.15 & [32.13-48.42] \\
\hline Knows transmission route & $0(0)$ & - & - \\
\hline Deworm their dogs regularly & $82(58.57)$ & 4.17 & [50.14-66.52] \\
\hline
\end{tabular}




\section{Discussion}

Toxocara canis, Ancylostoma caninum, Dipylidium caninum, and Echinococcus granulosus are well-known zoonotic parasites worldwide, resulting in high public health risks (Moskvina and Ermolenko, 2016). This study revealed the presence of four species of endoparasites with overall prevalence of 59.24\% (95\% CI: $52.84-65.35$ ) in owned dog population of Bishoftu town. This relatively high prevalence is recorded despite regular deworming at least twice per year being practiced as reported by 58.57\% (95\% CI: 50.14-66.52) of the interviewed dog-owning households of the town. This indicates the inadequacy of twice-yearly treatments to control dog parasitism effectively in Bishoftu signifying the need to increase treatment frequency. Comparatively, similar overall infection prevalence reported by previous studies conducted in Ethiopia (Hailu et al., 2007; Zewdu et al., 2010). The overall prevalence observed in this study is also in concordance with previous reports of other countries (Cardoso et al., 2014; Quyen et al., 2015). However, the overall prevalence recorded in this study is much lower than the previously reported prevalence that ranged from 73 to $90.7 \%$ in some parts of Ethiopia (Jones et al., 2011; Abere et al., 2012; Paulos et al., 2012; Gugsa et al., 2015; Merga and Sibhat, 2015; Tamerat et al., 2015). A recent review on canine helminths in sub-Saharan Africa revealed high pooled prevalence of 71\% (95\% CI: 63-79\%) across 36 studies (Chidumayo, 2018). A study conducted in Canada reported an overall infection prevalence of $16.5 \%$ (Joffe et al., 2011). These differences might be attributable to local variations such as different climatic conditions; different population structure of studied dogs and sample size. Moreover, it might be due to different levels of public awareness on the effect of these parasites on the health of dogs and humans, ease of access to veterinary services, awareness about the availability of anthelminthic drugs to treat dogs and lack of knowledge in the transmission routes. Regional variation in the prevalence of canine intestinal parasitism was well documented (Little et al., 2009; Moskvina, and Ermolenko, 2016).

In this study, the recorded prevalence of each of these gastrointestinal zoonotic helminths parasites of dogs were $33.61 \%$ Ancylostoma caninum, $29.41 \%$ Toxocara canis, $19.75 \%$ Dipylidium caninum, and $2.10 \%$ Echinococcus granulosus. The prevalence of Ancylostoma caninum and Toxocara canis was significantly higher $(\mathrm{p}<0.001)$ than the prevalence of Dipylidium caninum and Echinococcus granulosus. There is no single diagnostic technique capable of detecting all kinds of parasitic species present in dog feces (Mateus et al., 2014). The lower detection of Dipylidium caninum and Echinococcus granulosus egg might be 
due to the poor sensitivity of floatation technique for taeniidae egg detection. Using flotation technique, tapeworm eggs detected when free in the feces. However, tapeworm eggs passed from the host being contained in tapeworm segments and rarely found free in the feces. Therefore, fecal flotation tends to be a poor detector of tapeworm infection status (Zajac and Conboy, 2012). Less than 1\% detection of Taeniidae and Dipylidium caninum eggs was reported in faecal samples analyzed using sedimentation/flotation techniques compared to higher than 7\% detection of Ancylostoma spp. and Toxocara spp. (Mateus et al., 2014; Kostopoulou et al., 2017).

The infection prevalence of Ancylostoma caninum and Toxocara canis was significantly higher $(\mathrm{p}<0.001)$ in male than in female dogs. This is in agreement with the previous reports (Ramirez-Barrios et al., 2004; Maria et al., 2006; Davoust et al. 2008; Zewdu et al., 2010). This could be attributable to hormonal factors and sex-associated behaviors such as roaming and aggressive temperament possibly due to testosterone (Kirkpatrick, 1988). Studies have also demonstrated movement behavior frequently observed in male dogs. Androgens or male sex hormones, such as testosterone have a number of behavioral influences in adult males contributing to manifestation of sexually dimorphic behaviors, which are behaviors more common in one sex than in the other. Sexually dimorphic behaviors manifested in male dogs include roaming away from home, urine or scent marking, aggressiveness and fighting with other male dogs. Castration reduced roaming in 90\% of dogs through reduction of testosterone production without affecting behaviors that are similar between males and females such as hunting, playfulness, house guarding and seeking affection (Hopkins et al., 1976). In a demographic study conducted on a stray dog population in India, higher prevalence of male stray dogs of $>3$ months old age group was observed yielding a male-biased sex ratio of 1.3:1 to 1.4:1 (Totton et al., 2010). The male-biased sex ratio in roaming dog population might be attributed to the choice of male dogs as pets by the society. Most people generally prefer male dogs for their aggressive temperament to be used as guardian. In contrary, most people tend to avoid having female dogs due to the nuisance of a bitch in estrus attracting groups of intact males and some people hate to deal with unwanted puppies (Totton et al., 2010; Cortez-Aguirre et al., 2018).

Ancylostoma caninum was the most prevalent intestinal helminth parasite of dogs identified in this study. The predominance of Ancylostoma spp in dog fecal examination has been documented in prior studies reported from various 
geographical locations in Ethiopia (Hailu et al., 2007; Zewdu et al., 2010; Abere et al., 2012; Getahun and Addis, 2012; Gebreselasie et al., 2013; Tamerat et al., 2015), USA and Canada (Little et al., 2009; Joffe et al., 2011). The reported prevalence by previous studies from different geographic locations of Ethiopia ranged from 40-70\% in necroscopic study (Hailu et al., 2007; Zewdu et al., 2010; Merga and Sibhat, 2015). Ancylostoma spp. were also among the most commonly identified intestinal parasites with reported prevalence of $2.5 \%$ in the United States (Little et al., 2009) and 0.81\% in Canada (Joffe et al., 2011). These differences in prevalence of Ancylostoma spp. infection in dogs of different localities could be attributed to variations in the diagnostic technique employed, deworming practices, level of awareness and ecological factors. The studies that had reported prevalence of $50 \%$ and $70 \%$ from Ethiopia have used necropsy methods for diagnosis.

Toxocara canis is the second most prevalent zoonotic helminth parasites of dogs found in $29.41 \%$ of 238 dogs examined in this study. This is in agreement with the reported prevalence (25-32\%) from some parts of Ethiopia (Paulos et al., 2012; Gebreselasie et al., 2013; Abere et al., 2013; Tamerat et al., 2015)), but higher than the previous reported prevalence of $21 \%$ from Bishoftu (Hailu et al., 2007) and $11.72 \%$ from Adama (Merga and Sibhat, 2015). This indicates that the prevalence Toxocara canis has increased from the previous study conducted 10 years earlier in Bishoftu. Similar prevalence (30.0-35.7\%) with this study reported from Bangladesh (Shubhagata et al., 2012). Nevertheless, high incidence of Toxocara infection is reported from other developing countries (Dutta, 2002; Traub et al., 2002; Shubhagata et al., 2012; Fang et al. 2015). Toxocariasis is one of the most common parasitic zoonoses in the world capable of causing visceral and ocular larva migrans in humans, particularly in children. Routine year-round use of monthly anthelmintic to deworm regularly dogs significantly reduces prevalence of these parasites (Little et al., 2009; Joffe et al., 2011).

Dipylidium caninum is an intestinal parasite of domestic dog, domestic cat, wild carnivores and occasionally humans (East et al., 2013; Fang et al., 2015). D. caninum was the third most prevalent parasite purged from $19.41 \%$ of examined dogs after administration of praziquantel in this study. This result is in agreement with the prevalence of 22.4-23.7\% reported from some parts of Ethiopia (Getahun and Addis, 2012; Merga and Sibhat, 2015). The prevalence of this parasite recorded in this study is much higher than the prevalence of $6.56 \%$ reported from northern part of Ethiopia (Negash et al., 2014) and 2.3\% 
from Harar (Tamerat et al., 2015) indicating variation in the prevalence of $D$. caninum in different geographical areas of the country. This variation could be associated with variation in the diagnostic techniques used, the study population and the geographical area affecting the distribution of flea intermediate hosts that may be present relatively in a higher density in feral dogs than in owned dog population. Although infections with $D$. caninum are rare, it is a zoonotic parasite and humans, especially children can acquire the infection by accidentally ingesting infected fleas due to their playing habits and proximity with pet dogs and cats (Jiang et al., 2017).

Echinococcus granulosus was the least detected parasite in owned dog population in this study. The recorded low prevalence of $2.10 \%$ (5/238) E. granulosus in this study might be due to the low sensitivity of the fecal egg detection method used in this study. Similar low prevalence (3.6-6\%) reported by previous studies from Ethiopia using similar diagnostic method (Paulos et al., 2012; Gebreselasie et al., 2013; Gugsa et al., 2015; Merga and Sibhat, 2015). The prevalence $E$. granulosus is relatively low in reports from other parts of the world (Kostopoulou et al., 2017). This is partly associated with limitations of microscopic detection of $E$. granulosus eggs in fecal samples by routine coprological methods that suffers from a low sensitivity (Deplazes et al., 1992) and even the sensitivity of arecoline hydrobromide purgation may be as low as $39 \%$ for E. granulosus (Ziadinov et al., 2008). The other factor that might have contributed to the low detection rate of eggs is the intermittent release of eggs by the parasite (East et al., 2013). However, higher prevalence (17.3-61.5\%) was reported using necropsy as a diagnostic method (Zewdu et al., 2010; Jones et al., 2011). Comparative study conducted in Brazil and Ethiopia showed variability in the sensitivity of diagnostic tests, necropsy being more sensitive than coprology (Hailu et al., 2007; Klimpel et al., 2010) attributed to inability of coprological technique to detect immature parasites that do not lay eggs.

From a total of 469 households found registered within the territories of 10 Bishoftu town blocks randomly selected for this study, 334 (71.2\%) owned one or more dog. Of all households who responded to the questionnaire, $61.43 \%$ had single dog while the other $38.57 \%$ had two or more dogs. The questionnaire revealed $90 \%$ of the respondents keep dogs to guard them against thieves and wildlife whereas only $10 \%$ of the dog-owning population keeps dogs as companion animals. Dogs play a diversity of roles in different societies, cultures, social interests, religious convictions and occupation (Macpherson, et al., 2005). 
Households keep dogs for a purpose and are over-concerned for their safety. In this study, some households that had a previous negative experience from regular practice of strychnine-baited killing of roaming dogs by town's municipality became suspicious of the drug given for deworming might be lethal and refused to participate. Some households agreed to deworm their dogs but failed to submit fecal sample due to releasing their dogs to free roam and inability to observe where their dog defecated.

Owners often observe tapeworm segments in the perianal area or in feces (Zajac and Conboy, 2012). Around half of participants in this questionnaire survey have also observed a parasite in their dogs' excrement and knew that dogs can have parasites, but none of them has information about routes of transmission to humans indicating a need for public awareness creation to prevent potential exposure of people to dog borne parasitic zoonosis. Similar questionnaire survey conducted in Jimma reported that 19.4\% (34/175) of the respondents of that study had noticed parasitic disease in dogs, but had no information whether it can be transmitted to humans or not. The dog-owning population included in this study has relatively better awareness about potential transmissibility of dog parasites to humans than the reported $0 \%$ from Ambo by Zewdu et al. (2010), 3\% from Hawassa by Gebreselasie et al. (2013), 22\% from Wondoganet by Jones et al. (2011) and $27.1 \%$ from Harar by Tamerat et al., (2015). The current study population has better awareness as compared to the lower level of awareness reported by previous studies conducted in other geographic areas of Ethiopia. This might be due to exposure to and existence of long years of veterinary services in Bishoftu town that might have created relatively better awareness about the importance of animal treatment and ease of access to anti-helminthic drugs used for treatment of dogs and other animal species. This is also partly attributable to the awareness of the population about animal health and welfare created through National Veterinary Institute, Animal Welfare Projects and Veterinary Teaching Hospital of Addis Ababa University College of Veterinary Medicine and Agriculture as well as the existence of better access to formal education and relatively better living standard of the people.

This study indicates the existence of serious environmental pollution due to contamination by dog fecal material. More than one-third of dog owners either let their dogs to defecate in the public fields or dispose their dogs' excreta out of their compound. Nearly $90 \%$ of the respondent's clean dog excreta without 
wearing glove, facemasks, boots and/or coverall /gown indicating high risk of exposure to potentially dangerous zoonotic parasitic infection such as $E$. granulosus. The assessment of feeding practice also showed that more than $80 \%$ of the dog owners either feed uncooked offal from butcher houses or backyard slaughter or release their dogs to search for their own feed with a potential access to infected raw condemned offal. The practice of feeding raw or improperly cooked uninspected offal to dogs may increase the risk of public exposure since it could serve as a source of infection and perpetuate many parasitic zoonoses.

\section{Limitations of the study}

We have used flotation techniques for parasitic egg detection and collected fecal materials as well as expelled adult parasites 24 hours after treatment with ivermectin and praziquantel. All $D$. caninum detections except in two cases were based on expelled adult parasite, but we did not have record and do not remember the numbers of parasite-based detection versus egg-based detection separately for others parasites. The reported detection of $E$. granulosus egg solely based on egg detection might be an underestimation of the true prevalence. The questionnaire response rate was around 59\% and needs caution to generalize the interpretation as those who declined to respond may vary from those who responded in wealth status and educational level.

\section{Conclusion and recommendations}

The finding of this study revealed that zoonotic intestinal helminth parasites remain common in owned dogs of Bishoftu town. This indicates the existence of potential threat to the health of affected dogs and the public as well. Despite the zoonotic importance of these parasites, the dog-owners awareness and practice to protect themselves and the society from potential zoonotic infection of these parasites is at low level. Therefore, it is recommended to conduct a systematic study of these parasites on humans and the free-roaming dog population to elucidate the true extent of the problem in the dog population and the public to take appropriate actions towards awareness creation of dog-owning population and promote dog-deworming programs and to reduce the public's exposure. Following treatment with anthelminthic drugs used in this study, many dogs expelled adult parasites in their feces except for $E$. granulosus. Thus, future research needs to objectively evaluate the importance of anthelminthic treatment in improving diagnostic sensitivity to helminthic infection in dogs. 


\section{Acknowledgments}

The Addis Ababa University, Office of the Vice President for Research and Technology Transfer, which has funded this research and dog owning community who allowed deworming and subsequently submitted the fecal samples for laboratory diagnosis are sincerely acknowledged.

\section{References}

Abere, T., Bogale, B., and Melaku, A. 2013. Gastrointestinal helminth parasites of pet and stray dogs as a potential risk for human health in Bahir Dar town, northwestern Ethiopia. Vet.World. 6(7): 388-392.

Barutzki, D. and Schaper, R. 2003. Endoparasites in dogs and cats in Germany 1999 2002. Parasitol. Res., 90(3): S148-S150.

Bowman, D.D., Montgomery, S.P., Zajac, A.M., Eberhard, M.L., and Kazacos, K.R. 2010. Hookworms of dogs and cats as agents of cutaneous larva migrans. Trends Parasitol., 26(4): 162-167.

Cardoso, A.S., Costa, I.M.H., Figueiredo, C., Castro, A.; Conceicao, M.A.P. 2014. The occurrence of zoonotic parasites in rural dog populations from Northern Portugal. J. Helminthol., 88(2): 203-209.

Chidumayo, N.N. 2018. Epidemiology of canine gastrointestinal helminths in sub-Saharan Africa. Parasit. \& Vect., 11:100, https://doi.org/10.1186/s13071-018-2688-9

Cortez-Aguirre, G. R., Jiménez-Coello, M., Gutiérrez-Blanco, E., and Ortega-Pacheco, A. 2018. Stray Dog Population in a City of Southern Mexico and Its Impact on the Contamination of Public Areas. Hindawi Vet. Med. Int., https://doi. org/10.1155/2018/2381583.

Davoust, B., T. Normand, O. Bourry, H. Dang, E. Leroy, and G. Bourdoiseau. 2008. Epidemiological survey on gastro-intestinal and blood-borne helminths of dogs in North-East Gabon. Onderstepoort J. Vet. Res., 75: 359-364.

Deplazes, P., Gottstein, B., Eckert, J., Jenkins, D.J., Ewald, D., Jimenezpalacios, S., 1992. Detection of Echinococcus coproantigens by enzyme-linked immunosorbent assay in dogs, dingoes, and foxes. Parasitol. Res., 78(4): 303-308.

Dowd, K., Taylor, M., Toribio, J.L.M.L., Hooker, C. and Dhand, N. K. 2013. Zoonotic disease risk perceptions and infection control practices of Australian veterinarians: Call for change in work culture, Prev. Vet. Med., 11(1-2): 17-24.

Dutta, J.K. 2002. Disastrous results indigenous methods rabies prevention in developing countries. Int. J. Infect. Dis., 6: 236.

Ethiop. Vet. J., 2020, 24 (1), 93-115 
East, M.L., Kurze, C., Wilhelm, K., Benhaiem, S., Hofer, H., 2013. Factors influencing Dipylidium sp. infection in a free-ranging social carnivore, the spotted hyaena (Crocuta crocuta). Int. J. Parasitol. Parasites Wildl 2: 257-265.

Elsheikha, H.M., and Patterson, J.S. 2013. Self-Assessment Colour Review Veterinary Parasitology. Manson Publishing Ltd, London, UK, pp 256.

Fang, F., Li, J., Huang, T., Guillot, G., and Huang, W., 2015. Zoonotic helminths parasites in the digestive tract of feral dogs and cats in Guangxi, China. BMC Vet. Res. 11:211

Finsterer, J. and Auer, H. 2013. Parasitoses of the human central nervous system. J. Helminthol., 87(3): 257-270.

Fromsa, A. and Jobre, Y. 2012. Estimated annual economic loss from organ condemnation, decreased carcass weight and milk yield due to bovine hydatidosis (Echinococcus granulosus, Batsch, 1786) in Ethiopia. Ethiop. Vet. J., 16 (2):1-14

Gawor, J., Borecka, A., Zarnowska, H., Marczynska, M. and Dobosz, S. 2008. Environmental and personal risk factors for toxocariasis in children with diagnosed disease in urban and rural areas of central Poland. Vet. Parasitol., 155(3-4): 217-222.

Gebreselasie, D., Geyola, M., Dagne, E., Asmare, K. and Mekuria, S. 2013. Gastrointestinal Helminthes in Dogs and Community Perception on Parasite Zoonosis at Hawassa City, Ethiopia. Glob. Vet., 11 (4): 432-440.

Getahun, Z. and Addis, M. 2012. Prevalence of gastrointestinal helminthes among dogs in Bahir Dar town, Ethiopia. World Appl. Sci. J., 19 (5): 595-601.

Goggle Map, http://earth.google.com/streetview/, accessed on December 20, 2017.

Gugsa, G., Hailu, T., Kalayou, S., Abebe, N. and Hagos, Y. 2015. Study on gastro-intestinal helminth parasites of dogs in Mekelle city, Tigray Ethiopia. J. Parasitol. Vect. Biol. 7(2): 26-36.

Hailu, Y.., Taddese, A.., Regassa, F., and Basu, A.K. 2007. Gastrointestinal nematodes in dogs from Debre Zeit, Ethiopia. Vet. Parasitol., 148(2): 144-148.

Hendrix, C.M. 2003. Laboratory procedures for veterinary technicians, $4^{\text {th }}$ edition, Mosby publishing, USA, Pp 364 .

Hopkins, S.G., Schubert, T.A., and Hart, B.L. 1976. Castration of adult male dogs: effects on roaming, aggression, urine marking, and mounting. J. Am. Vet. Med. Assoc. 168 (12):1108-1110.

Jiang, P., Zhang, X., Liu, R.D., Wang, Z.Q. and Cui, J. 2017. A Human Case of Zoonotic Dog Tapeworm, Dipylidium caninum (Eucestoda: Dilepidiidae), in China. Kor. J. Parasitol., 55 (1): 61-64. 
Joffe, D., Niekerk, D. V., Gagné, F., Gilleard, J., Kutz, S., Lobingier, R., 2011. The prevalence of intestinal parasites in dogs and cats in Calgary, Alberta. Can. Vet. J., 52(12): 1323-1328

Jones, O., Kebede, N., Kassa, T., Tilahun, G., and Macias, C. 2011. Prevalence of dog gastrointestinal parasites and risk perception of zoonotic infection by dog owners in Wondo Genet, Southern Ethiopia. J. Pub. Hlth. Epidemiolo., 3 (11): 550-555.

Kirkpatrick, C.E., 1988. Epizootiology of endoparasitic infections in pet dogs and cats presented to a veterinary teaching hospital. Vet. Parasitol., 30(2): 113-124.

Klimpel, S., Heukelbach, J., Pothmann, D., and Ruckert, S. 2010. Gastrointestinal and ectoparasites from urban stray dogs in Fortaleza (Brazil): High infection risk for humans? Parasitol. Res., 107(3): 713-719.

Kostopoulou, D., Claerebout, E., Arvanitis, D., Ligda, P., Voutzourakis, N., Casaert, S. and Sotiraki, S. 2017. Abundance, zoonotic potential and risk factors of intestinal parasitism amongst dog and cat populations: The scenario of Crete, Greece. Parasit \& Vectors., 10:43 DOI 10.1186/s13071-017-1989-8.

Little, S.A., Johnson, E.M., Lewis, D., Jaklitsch, R.P., Payton, M.E., Blagburn, B.L., et al., 2009. Prevalence of intestinal parasites in pet dogs in the United States. Vet. Parasitol. 166(1-2): 144-152. doi: 10.1016/j.vetpar.2009.07.044.

Lorenzini, G., Tasca, T., and De Carli, G.A. 2007. Prevalence of intestinal parasites in dogs and cats under veterinary care in for to Alegre, Rio Grande do Sul, Brazil. Braz. J. Vet. Res. Anim. Sci. 44(2): 137-145.

Macpherson, C.N.L. 2005. Human behaviour and the epidemiology of parasitic zoonoses, Int. J. Parasitol., 35(11-12):1319-1331

Maria, F., Fontanarrosa, V., Dar'O, B., Julia, B., and Diego, E. 2006. An epidemiological study of gastrointestinal parasites of dogs from Southern Greater Buenos Aires (Argentina): Age, gender, breed, mixed infection and seasonal and spatial patterns. Vet. Parasitol., 136(3-4): 283-295.

Mateus T. L., Castro, A., Ribeiro, J. N., and Vieira-Pinto, M. 2014. Multiple Zoonotic Parasites Identified in Dog Feces Collected in Ponte de Lima, Portugal - A Potential Threat to Human Health. Int. J. Environ. Res. Pub. Hlth., 11(9): 9050-9067, doi:10.3390/ijerph110909050.

Mengistu, S., Keftasa, D. and Yami, A. 2002. Productivity of four Sesbania species on two soil types in Ethiopia. Agroforest. Syst. 54: 235-244.

Merga, T., and Sibhat, B. 2015. Prevalence of gastrointestinal helminth parasites of dogs and associated risk factors in Adama Town, Central Ethiopia. Ethiop. Vet. J., 19 (2): 91-103.

Ethiop. Vet. J., 2020, 24 (1), 93-115 
Mirzaei, M., and Fooladi, M. 2013. Coproscopy survey of gastrointestinal parasites in owned dogs of Kerman city, Iran. Vet. Ital., 49(3): 309-13.

Morey, D.F., 1994: The early evolution of the domestic dog. Am. Sci., 82(4): 336-347.

Moskvina, T. V., and Ermolenko, A. V. 2016. Helminth infections in domestic dogs from Russia. Vet. World, 9 (11): 1248-1258.

Negash, G., K/Yohannis, T., Afera, B., Mengiste, B., Weldu, K. and W/Mariam H.S. 2014. Zoonotic Importance and Prevalence of Parasites in Dogs: Pet Owner's Knowledge Assessment. Euro. J. Appl. Sci., 6 (3): 45-49.

Paul, A.M., Overgaauw, Linda-van, Z.B., Denise, H., Felix, O., Yaya, J.R., Elena, P., et al., 2009. Zoonotic parasites in fecal samples and fur from dogs and cats in The Netherlands. Vet. Parasitol., 163(1-3): 115-122.

Paulos, D., Addis, M., Fromsa, A. and Mekbib, B. 2012. Prevalence of gastrointestinal helminths among dogs and owner's perception about zoonotic dog parasites in Hawassa Town, Ethiopia. J. Pub. Hlth. Epidemiol., 4 (8), 205-209.

Quyen, N.T., Lan, N.T.K, Van, C. and Nang, N.T. 2015. A Study on Prevalence of Intestinal Nematodes in Dogs in Phutho Province. J. Agri. Tech., 11 (8): 2563-2576.

Ramirez-Barrios, R.A., Barboza-Mena, G., Munoz, J., Angulo-Cubillán, F., .and Hernandez, E. 2004. Prevalence of intestinal parasites in dogs under veterinary care in Maracaibo, Venezuela. Vet. Parasitol., 121(1-2): 11-20.

Shubhagata, D.A.S., Abdul A.L., Suchandan, S.I.K. and Aungshuman, D. G.U. 2012. Prevalence and Worm Load of Enteric Helminthiasis in Stray Dogs of Chittagong Metropolitan, Bangladesh. YYU Vet Fak. Derg., 23 (3): 141 - 145.

Tamerat, N., Shimelis, D., Dagim, N. and Terefe, Y., 2015. Gastrointestinal parasites of pets and zoonosis awareness assessment of owners in Harar Town, Eastern Ethiopia. World. Appl. Sci. J., 33 (8): 1348-1354,

Totton, S. C., Wandeler, A. I., Zinsstag, J., Bauch, C. T., Ribble, C. S., Rosatte, R.C., McEwen, S.A. 2010. Stray dog population demographics in Jodhpur, India following a population control/rabies vaccination program. Prev. Vet. Med., 97(1): 51-57. doi: 10.1016/j.prevetmed.2010.07.009.

Traub, R.J., Robertson, I.D., Irwin, P., Mencke N., Monis, P. and Thompson, R.C. 2002. Humans, dogs, and parasitic zoonoses - unraveling the relationships in a remote endemic community in northeast India using molecular tools. Parasitol. Res., 90: 5156-5157.

Westgarth, C., Boddy, L. M., Stratton, G., German, A.J., Gaskell, R.M. and Coyne, K.P., 2013. Pet ownership, dog types and attachment to pets in 9-10-year-old children in Liverpool, UK. BMC Vet. Res., 9: 11-86. doi: 10.1186/1746-6148-9-102. 
Zajac, A. M., and Conboy, G. A. 2012. Veterinary Clinical Parasitology, $8^{\text {th }}$ edition John Wiley \& Sons, Inc., Ames, Iowa, USA, Pp 354

Zewdu, E., Semahegn, Y. and Mekbib, B. 2010. Prevalence of helminth parasites of dogs and owner's awareness about zoonotic parasites in Ambo town, Central Ethiopia. Ethiop. Vet. J., 14(2): 17-30.

Ziadinov, I., Mathis, A., Trachsel, D., Rysmukhambetova, A., Abdyjaparov, T.A., Kuttubaev, O.T., Deplazes, P. and Torgerson, P.R. 2008. Canine echinococcosis in Kyrgyzstan: using prevalence data adjusted for measurement error to develop transmission dynamics models. Int. J. Parasitol. 38(10):1179-1190. doi: 10.1016/j. ijpara.2008.01.009. 